\title{
Rheopheresis in vascular diseases
}

\author{
Melinda Vass ${ }^{\mathrm{a}, 1}$, Ágnes Diószegi ${ }^{\mathrm{a}, 1}$, Norbert Németh ${ }^{\mathrm{b}}$, Viktória Sógor ${ }^{\mathrm{b}}$, Sándor Baráth $^{\mathrm{c}}$, \\ Eszter Szalai ${ }^{\mathrm{d}}$, László Módis ${ }^{\mathrm{d}}$ and Soltész Pál ${ }^{\mathrm{a}, *}$ \\ ${ }^{a}$ Department of Internal Medicine, Division of Angiology, University of Debrecen Clinical Center \\ ${ }^{\mathrm{b}}$ Department of Operative Techniques and Surgical Research, University of Debrecen Clinical Center \\ ${ }^{\mathrm{c}}$ Department of Laboratory Medicine, University of Debrecen Clinical Center \\ ${ }^{\mathrm{d}}$ Department of Ophtalmology, University of Debrecen Clinical Center
}

\begin{abstract}
Rheopheresis is an extracorporal selective double-filtration procedure. In the first part of the treatment the blood is passes through the plasma filter, which separates blood cells from the plasma. Then the plasma flow to a second filter called MONET (Membranefiltration Optimised Novel Extracorporal Treatment). The MONET filter retains high molecular weight proteins such LDL, Lp(a), fibrinogen, $\alpha 2$ macroglobulin, vWF and IgM. Hereby the whole blood and plasma viscosity decrease, improves microcirculation, and has a positive effect on lipid profile as well.

Accorging to ASFA recommendation rheopheresis is a first line treatment in age-related dry macular degeneration and in sudden sensorineural hearing loss. There are other clinical situations in which rheopheresis has been used effectivly. But only few data are available and large clinical trials have not been done in these diseases. In this paper we describe a case history and laboratory findings of a patient who suffers from age related dry macular degeneration and was successfully treated by rheopheresis.
\end{abstract}

Keywords: Rheopheresis, microcirculation, age-related dry macular degeneration, anti-inflammatory effect, haemorheological parameters

\section{Introduction}

During the past few years several novel therapeutic apheresis methods were introduced, significantly widening the indication range of apheresis therapies. One of these new therapeutic procedures is called rheopheresis, which is classified into the group of selective extracorporal haemotherapies. Rheopheresis is a 2-step cascade filtration procedure [20]. As the first step of the treatment cellular elements are separated from the plasma, then the plasma flows through a special filter which is able to eliminate certain plasma components based on molecular size, or at least it is able to reduce their level significantly [3].

With the help of the MONET filter (Membrane filtration Optimised Novel Extracorporal Treatment) developed by Fresenius SE (Bad Homburg, Germany) the opportunity to perform a rheopheresis is provided. With this method proteins with molecular weights higher than $250-300 \mathrm{kDa}$ can be eliminated from the plasma. After that the purified plasma is administered to the patient through a peripheral vein, together with the cellular elements. LDL, Lp(a), fibrinogen, $\alpha 2$ macroglobulin, vWF and IgM are some of those proteins of high molecular weight which are eliminated this way. Averagely, 1 treatment reduces the LDL level by approximately $70 \%$, the total cholesterol level by $50 \%$, and it decreases the triglyceride level generally by $35-40 \%$. The fibrinogen level is also reduced to its half in general, after one treatment. On the other hand it needs to be highlighted that HDL level does not change significantly, neither do plasma albumin nor total protein levels [19].

\footnotetext{
${ }^{1}$ Theses authors are Equal contributed: Dr. Vass Melinda, Dr. Diószegi Ágnes.

*Corresponding author: Prof. Dr. Pál Soltész, Division of Angiology, Clinical Center, University of Debrecen, 4032 Debrecen, Móricz Zs. 22. E-mail: dr.soltesz.pal@gmail.com.
} 
Besides the above mentioned facts they also observed that rheopheresis is able to decrease $\operatorname{Lp}(\mathrm{a})$, vWF and IgM levels as well. The favourable effect of the rheopheresis therapy is resulting from the beneficial changes in levels of these macromolecules. As a result of this, treatment viscosity of the plasma and the whole blood is reduced, the erythrocyte aggregation is moderated and deformability of the erythrocytes is improved [3,22].

As a consequence of these, blood flow is improved, and dysfunctions of the microcirculation are also alleviated. It has a beneficial effect on lipid profile as well, it decreases the level of atherogenic lipid fractions in blood, but at the same time it does not significantly alter the levels of protective HDL.

Indication range of rheopheresis has been widened during the past few years. They conducted trials with rheopheresis in different disease groups, however, randomized, prospective clinical trials were only conducted in case of age-related macular degeneration and sensorineural hearing loss $[4,17]$. Rheopheresis was applied in case of patients with peripheral arterial vascular diseases, who were not suitable for revascularization or who suffered from diabetic leg syndrome [9]. Futhermore beneficial effects were detected on the blood flow in the carotid artery and on the vasodilatory capacity of the coronary arteries [6].

Based on double blind, placebo-controlled trials, and 2013 guidelines of the American Society for Apheresis (ASFA), rheopheresis is the recommended first choice of therapy in case of dry form of age-related macular degeneration.

Age-related macular degeneration (AMD) is a special angiological disease, which has two different types, dry and exudative form. About $80 \%$ of all AMD cases are dry forms. It shows the significance of AMD that based on a WHO survey it is responsible for $50 \%$ of ophthalmological diseases leading to blindness in Western countries, and the ratio is similar in Hungary [15]. The disease shows direct correlation with cholesterol, fibrinogen and alpha-macroglobulin levels. Its pathomechanism is multi-factorial, in which several different mechanisms pay role, such as inflammatory involvement of chorio-capillaries, lipid accumulation, haemorheological disorders and dysfunctions of microcirculation. Among the subjective symptoms we have to outline that the patient initially sees a blurred area, which is always in the way of sight (central visual impairment). Currently there are no available causal treatments, but progression can be slowed down with conservative therapy and moderation of the risk factors. In case of dry form of AMD we can administer antioxidant vitamins, zinc, lutein, or zeaxantin containing dietary supplements, as well as nutrients rich in unsaturated fatty acids, and in case of the exudative form of AMD, intra-vitreal pharmacotherapy (VEGF inhibitors) is recommended [1-2, 14].

\section{Case report}

In the medical anamnesis of our 68-year-old male patient, medical care due to hypertension and asthma, as well as TIA is listed. Considering his risk factors, he has been a heavy smoker for 30 years, but he quit smoking in 2009. No ophthalmological diseases are present in familial medical anamnesis.

His eye-related complaints started to emerge during summer of 2013 he experienced blurred vision and central visual field loss. Based on the dilated fundus examination, optical coherence tomography (OCT), and fluorescent angiography we diagnosed dry type age-related macular degeneration in the background of his complaints. In line with the most intensive conservative therapy (zinc and lutein containing dietary supplements) his visual loss progressed continuously. By the spring of 2014, his visual impairment made him unable to read. Because of the rapidly progressing condition-despite the conservative therapy - we decided to perform a rheopheresis treatment together with the Department of Ophthalmology. Before we initiated the therapy we obtained a licence from the local Ethics Committee and the written informed consent form signed by the patient. 
The first rheopheresis treatment was performed in July 2014 at the ICU of our clinic. Between July 2014 and July 2016 the patient received 6 cycles of rheopheresis treatment, altogether for 11 times. The rheopheresis treatment was performed with the use of a MONET filter. No complications or side effects were observed during the course of the therapy.

For efficacy assessment of the rheopheresis treatment we performed laboratory tests and angiological examinations-besides the ophthalmological tests-preceding the treatment cycles, as well as at the end of each therapeutic cycle. According to our assumption, rheopheresis treatment not only works via improvement of viscosity to moderate microcirculatory dysfunction, but it may have other antiinflammatory effects, and it might decrease the level of endothelial and thrombocyte activating markers. All these beneficial effects may result in improving the endothelial functions.

\section{Rheopheresis}

The rheopheresis treatment was performed by using the MONET (Membrane filtration Optimised Novel Extracorporal Treatment) filter developed by Fresenius Medical Care Deutschland GmbH. The MONET filters were attached to Art Universal devices. For the extra-corporal treatment, cannulation of two peripheral veins is necessary. As first step of the therapy we separate the plasma from the cellular components of blood with the help of a plasma filter, then the plasma is made to flow through the MONET filter. After that the purified plasma is administered into the patient's circulatory system through a peripheral vein, together with the cellular elements. Citrate was administered as an anticoagulant.

\section{Haemorheological measurements}

Determination of the viscosity of the whole blood and plasma (WBV, PV [mPa.s]) was performed with a Hevimet-40 capillary viscometer (Hemorex Ltd., Hungary). Viscosity values at $90 \mathrm{~s}^{-1}$ shear rate were used for comparison. According to the Mátrai-formula, the whole blood viscosity data were also corrected to $40 \%$ hematocrit value $[8,12]$. Deformability of erythrocytes was measured by atational ektacytometer (LoRRca MaxSis Osmoscan, Mechatronics BV, The Netherlands). Elongation index (EI) was plotted in the function of shear stress (SS [Pa]). RBC aggregation was determined concomitantly with two different methods. First we used a Myrenne MA-1 erythrocyte aggregometer (Myrenne $\mathrm{GmbH}$, Germany), the operation of which is based on light transmission. Aggregation indices were determined under stasis (M mode) and at $3 \mathrm{~s}^{-1}$ shear rate (M1 mode), at the 5 th and the 10th second of the aggregation (M $5 \mathrm{~s}$, M $10 \mathrm{~s}$, M1 $5 \mathrm{~s}$, M1 10s). The LoRRca system characterizes the aggregation process based on light reflection (syllectometry). For comparison the following parameters were used: aggregation index \% (AI), amplitude (Amp), t1/2 [s].

\subsection{Laboratory tests}

Phagocyte activity was measured by chemiluminescence method. Heparinized human whole blood was used. Samples were diluted by PBS (phosphate buffered saline), in the ratio of $1: 3$. In the control tubes, $0.5 \mathrm{ml}$ of diluted blood was diluted to $1 \mathrm{ml}$ by adding $0.5 \mathrm{ml}$ of PBS. For the stimulation of phagocytes, $0.5 \mathrm{ml}$ of zymosan suspension ( $1 \mathrm{mg} / \mathrm{ml}$ zymosan, Sigma, MO, USA) was added. As positive control, phagocytes were stimulated by $0.5 \mathrm{ml}$ PMA $(0.04 \mu \mathrm{g} / \mathrm{ml}$, phorbol-myristate-acetate, Sigma). The amplification of free radical production (chemi-luminescence, CL) took place by the addition of $50 \mu \mathrm{l}$ luminol ( $20 \mu \mathrm{g} / \mathrm{ml}, 5$ amino-2,3 dihydro-1,4 phtalazinedione, Sigma). The CL was measured by an AutoLumat LB 953 multi-tube luminometer (Berthold Technologies, Germany). Both 
the non-stimulated controls (basal CL), the zymosan-stimulated and the PMA-stimulated whole blood samples were tested in duplicate. Their CL was detected in every 3 minutes for 45 minutes. Results are presented as the area under the relative-light unit (RLU) curves and the maximum values of RLUs.

Activated monocytes $(C D 14+C D 16+)$ were measured by flow cytometry from heparinized whole blood. Cells in $100 \mu \mathrm{l}$ blood were stained with 10-10 $\mu$ l of monoclonal antibodies (anti-CD16-FITC, anti-CD14-PE, Becton Dickinson, USA). Mouse IgG1-FITC, IgG1-PE (Becton Dickinson, USA) was used as an isotype control. After $25 \mathrm{~min}$ incubation the red blood cells were haemolysed and the leukocytes were washed with PBS (completed with BSA and sodium-azide). Finally the cells were suspended in $800 \mu \mathrm{l}$ of $1 \%$ para-formaldehyde. Samples were measured by a Beckman Coulter FC500 flow cytometer and analysis was carried out by CXP software (Beckman Coulter, USA). Monocytes were gated on the basis of their side-scatter parameter and CD14 positivity. Results were expressed as the percentage of CD16+ cells in the gated CD14+ monocyte region.

Determination of lipid profile (cholesterol, triglyceride, LDL, HDL, Lp(a) levels) was performed based on routine laboratory diagnostic tests at DE KK LMI (University of Debrecen, Clinical Centre, Institute of Laboratory Medicine). Analyses were performed from $4 \mathrm{ml}$ blood samples, free from anticoagulants. Determination of fibrinogen was done from blood sample treated with Na-citrate anticoagulant, according to the Clauss method. The reference range was: $1.5-4 \mathrm{~g} / \mathrm{L}$.

Measurement of $v W F$ :Ag level was performed from blood sample treated with Na-citrate anticoagulant. The whole blood was first centrifuged $\left(1500 \mathrm{~g}, 20 \mathrm{~min}, 22^{\circ} \mathrm{C}\right)$, then from the plasma obtained this way, level of vWF:Ag was determined by immuno-turbidimetry. The degree of agglutination is directly proportional to the level of vWF found in the sample. The reference range was between 50 and $160 \%$.

P-selectin expression characterising thrombocyte activation was determined from whole blood treated with citrate anticoagulant, with the help of anti-CD-62 monoclonal antibodies. Measurements were done by flow cytometer. Reference range of thrombocyte P-selectin was: 0-2\%.

Flow-mediated vasodilatation (FMD) was performed by ultrasound technic. Examinations were performed under standardized conditions on the patient, after 8 hours of fasting and 18 hours of smoking prohibition, on the right brachial artery. Measurements were made with Phillips (HD11XE) high resolution ultrasound device, with a $5-10 \mathrm{MHz}$ linear transducer. ECG monitoring was performed during the whole examination. We took longitudinal cross-sectional images of the brachial artery proximally from the elbow, and we determined the diameter of the artery between at least 5 identical points. Measurements were made synchronized to the R-wave. After that we triggered reactive hyperaemia, first by bloating the sphygmomanometer cuff placed onto the forearm for 4.5 minutes, maintaining a supra-systolic value $50 \mathrm{mmHg}$ above the systolic pressure, then suddenly deflating the cuff. We detected the change in the aortic diameter within the 60th second following the cuff deflation, accordingly to the method detailed above. The flow-mediated dilation was given as a percentage value, which expresses the diameter change triggered by reactive hyperaemia, compared to the resting diameter.

Arterial stiffness determination (augmentation index, pulse wave velocity) was done with the help of a Tensio Clinic arteriograph (TensioMed Kft., Debrecen). The measurement is based on the principle that as a result of contraction of the heart, the first pulse wave generated in the aorta is reflected at the level of bifurcation, thus an easily detectable second wave (late systolic peak) appears during systole. Morphology of the second wave depends on stiffness parameter of the common carotid artery, on the reflection time measured on the brachial artery at $35 \mathrm{mmHg}$ supra-systolic pressure (RT S35), as well as on peripheral resistance defined by the amplitude of the wave. The pulse wave velocity is the distance between the jugular fossa and the symphisis, and ratio of the RT S 35 value. Unit of the quotient obtained this way is $\mathrm{m} / \mathrm{s}$. 
Ophthalmological examination determined the best corrected visual acuity of the patient on a logarithmic scale, we took colour photos of the eye fundus (TRC-NW7SF, Topcon, Tokyo, Japan) and we performed an OCT (Zeiss Stratus, Carl Zeiss Meditec, Inc, California USA) examination.

\section{Results}

\subsection{Ophthalmological results}

Even the first cycle of therapy resulted in significant improvement in visual acuity. Reading ability of the patient was restored, he was able to read with a magnifier.

The objective best corrected visual acuity showed fluctuation, but no progression could be detected. Based on the examination of the eye fundus, the wasting of the right eye was not aggravated as a result of the therapy, and sub-retinal bleeding of the left eye absorbed completely by the end of the third cycle of therapy (Fig. 1). The OCT examination performed prior to the treatments showed extended retinal pigment epithelium (RPE) atrophy on the right side, pigment aggregation, thinned neuroretina and presence of sub-retinal fibro-vascular membrane in the left eye. In addition, pigment epithelial abruption distorting the fovea, and nasally from the centre, a small amount of sub-retinal fluid could be detected. By the end of the therapeutic cycles the contour of the fovea on the right became even, no progression was observed, neither intra-retinal nor sub-retinal fluid was visible on the left eye, and the pigment epithelium abruption also ceased (Fig. 2).

\subsection{Changes of haemorheological and lipid parameters as a result of rheopheresis}

Viscosity of whole blood and the plasma was increased prior to the first treatment, and increased erythrocyte aggregation was also measured, in line with borderline fibrinogen level. It can be observed that by the end of the first therapeutic cycle viscosity of the plasma returned to the normal range, viscosity of the whole blood also decreased significantly, it almost reached the reference range. Erythrocyte aggregation normalized and fibrinogen level also decreased. During the additional therapeutic cycles we observed obtained similar results (Table 1). In case of our patient lipid parameters were already within the normal range before initiation of the rheopheresis, due to the long-term statin therapy that he received because of secondary prevention of the formerly developing TIA. But still, our results prove that rheopheresis therapy was able to effectively decrease the level of atherogenic lipid fractions during the treatment cycles.

\subsection{Anti-inflammatory, thrombocyte and endothelial activation moderation effects of rheopheresis}

Prior to the first therapeutic cycle significantly increased phagocyte activity and increased CD14+/CD16+ cell population ratio were detected. Our results support the hypothetic inflammatory involvement of chorio-capillaries in pathogenesis of AMD. After the first treatment session, as a result of that phagocyte activity and CD14+/CD16+ cell population ratio normalized. Based on all these we assume that rheopheresis therapy has - besides the decrease in known macromolecular levels - other effects as well, for the detection of which further tests should be performed.

Rheopheresis treatment also moderated thrombocyte aggregation, which was evidenced by decrease and normalization of P-selectin expression. 
Table 1

Changes of laboratory parameters after rheopheresis treatments

\begin{tabular}{|c|c|c|c|c|c|c|c|c|c|c|c|c|}
\hline & \multicolumn{2}{|c|}{ Treatment period 1} & \multicolumn{2}{|c|}{ Treatment period 2} & \multicolumn{2}{|c|}{ Treatment period 3} & \multicolumn{2}{|c|}{ Treatment period 4} & \multicolumn{2}{|c|}{ Treatment period 5} & \multicolumn{2}{|c|}{ Tretament period 6} \\
\hline & Before & After & Before & After & Before & After & Before & After & Before & After & Before & After \\
\hline \multirow{2}{*}{$\begin{array}{l}\text { Whole Blood-Plasma } \\
\text { viscosity (mPa sec) }\end{array}$} & 6.28 & 4.34 & 4.25 & 4.22 & 5.1 & 4.8 & 5 & 4.19 & 4.44 & 4.1 & 4.57 & 4 \\
\hline & 1.55 & 1.17 & 1.25 & 1.19 & 1.38 & 1.18 & 1.47 & 1.3 & 1.45 & 1.35 & 1.67 & 1.67 \\
\hline Ert aggregability & Increased & Normal & Normal & Normal & Normal & Normal & Normal & Normal & Normal & Normal & Normal & Normal \\
\hline Fibrinogen $(\mathrm{g} / \mathrm{l})$ & 3.29 & 2.43 & 3.05 & 2.49 & 4.36 & 2.09 & 4.24 & 2.19 & 3.71 & 3.5 & 3.99 & 3.57 \\
\hline $\mathrm{Tg}(\mathrm{mmol} / \mathrm{l})$ & 0.8 & 0.6 & 0.5 & 0.4 & 0.7 & 0.9 & 0.7 & 0.7 & 0.7 & 0.6 & 0.8 & 0.7 \\
\hline Cholesterine (mmol/l) & 3.6 & 2.1 & 4.6 & 2.8 & 4.2 & 1.8 & 4.5 & 2.3 & 3.3 & 1.6 & 4.3 & 2.4 \\
\hline LDL-C (mmol/l) & 2.5 & 0.8 & 3 & 1.2 & 2.7 & 0.7 & 3 & 1.3 & 2.9 & 1.1 & 2.9 & 1.1 \\
\hline $\mathrm{Lp}(\mathrm{a})(\mathrm{mg} / \mathrm{l})$ & 141 & 69 & 135 & 69 & 60 & 32 & 80 & 58 & 62 & 42 & 62 & 42 \\
\hline $\begin{array}{c}\text { CD14+/CD16+ activated } \\
\text { monocyte ratio }(\%)\end{array}$ & 70.9 & 34.3 & 14.6 & 13.2 & 13.5 & 11.1 & 8.8 & 8.2 & 12 & 10 & 13 & 10 \\
\hline $\begin{array}{l}\text { Fagocyte activity } \\
\text { (RLU/30 min) }\end{array}$ & 12938 & 3452 & 525 & 422 & 2852 & & 127 & 115 & 43.8 & 108 & 104.9 & 111.8 \\
\hline P-selectin expression (\%) & 3.15 & 1.1 & 1.57 & 1.12 & 1.27 & 2.6 & 1.32 & 2.33 & 1.3 & 0.9 & 1.94 & 1.94 \\
\hline vWF:Ag (\%) & 114 & 90 & 107 & 89 & 116 & 86 & 121 & 81 & 124 & 110 & 131 & 80 \\
\hline
\end{tabular}



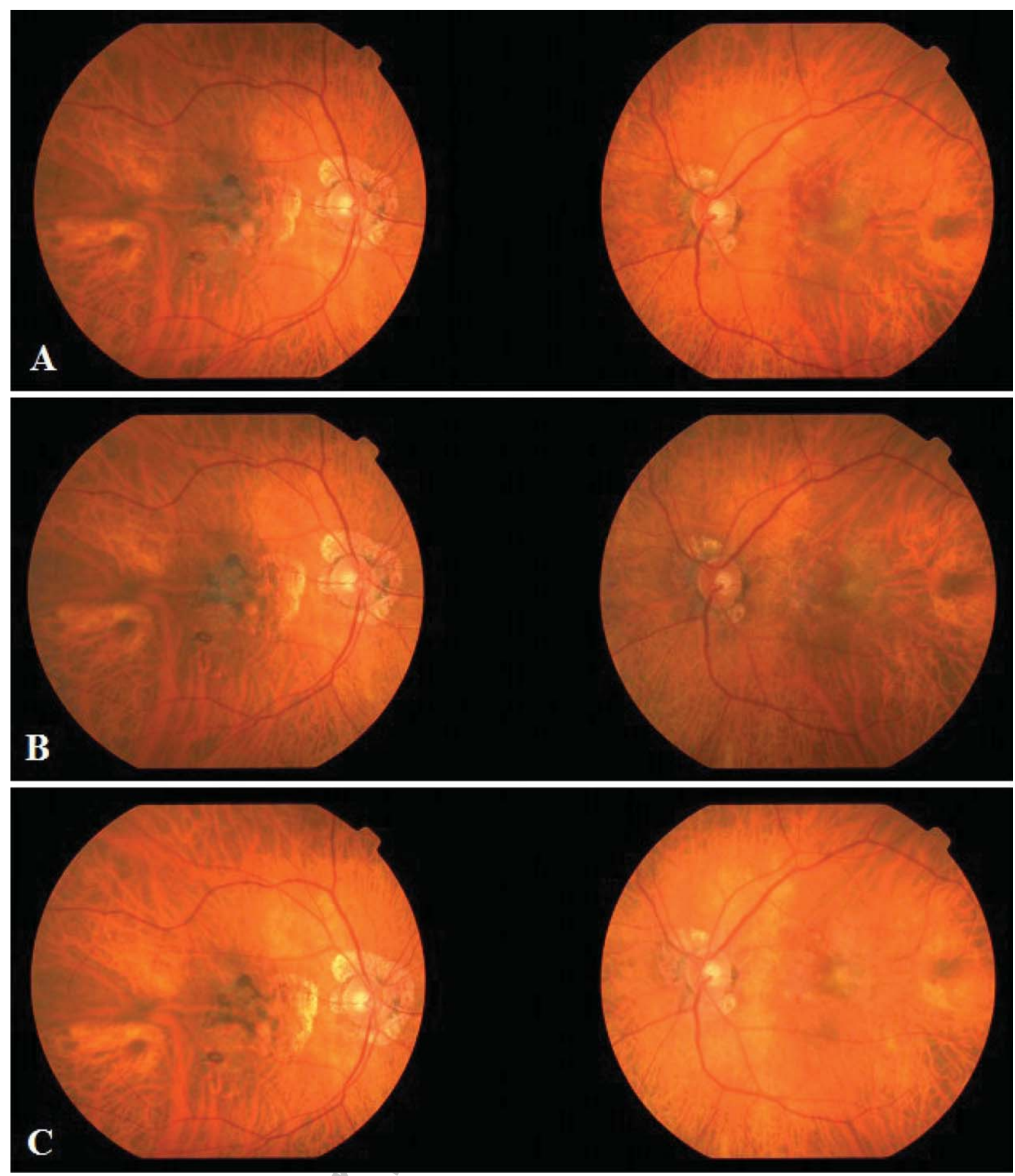

Fig. 1. Picture of the patient's coloured fundus: before the 1st treatment (A), after the 2nd (B), and after the 3rd treatment (C).

\subsection{Effect of rheopheresis on endothelial function and on stiffness parameters}

Beneficial effects emerging as a result of rheopheresis therapy may not only be exhibited via enhancing blood flow and improvement of microcirculation. According to our assumption these effects can 

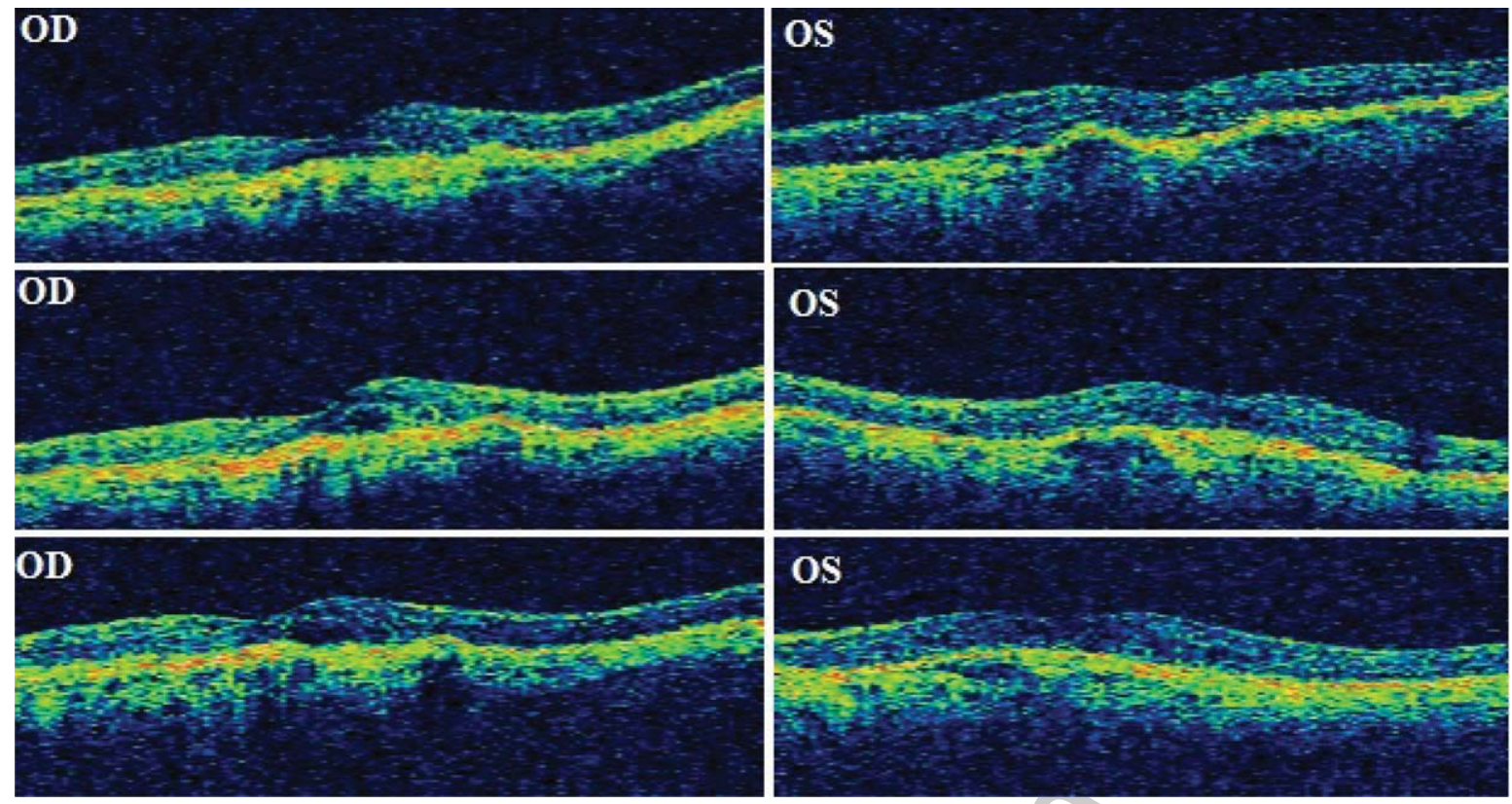

Fig. 2. OCT image: before the 1st rheoferezis treatment (on the top), after the 2nd treatment (in the middle) and after the 3rd treatment (below).

improve macrocirculation as well. Within the confines of this endothelial dysfunction may be improved, as well as stiffness parameters (Fig. 3a,b,c). Our results support the observation that resulting from the rheopheresis therapy endothelial dysfunction eased, and it also had a positive effect on vascular wall stiffness. Based on all these rheopheresis may have potential application in the treatment of vascular disease developing as a result of atherosclerosis.

\section{Discussion}

The age-related macular degeneration is a leading cause of severe visual impairment and blindness of the population above the age of 60 in developed countries. Vast majority, about $80 \%$ of all AMD cases are classified into the group of dry type.

The risk of developing the disease increases exponentially with ageing.

Smoking unambiguously affects the development and course of the disease, but alcohol consumption, obesity, pathological BMI can also be influencing factors.

Dry type AMD does not have a causal therapy currently. The following new strategies are available regarding the treatment of dry type AMD: a.) decreasing the development of drusen, b.) alleviating the inflammation, c.) decreasing the retinal oxidative stress and accumulation of toxic metabolites, $\mathrm{d}$. increasing the chorioidea perfusion e.) enhancing regeneration of RPE and photoreceptor cells, f. gene therapy [5].

Besides the above mentioned, rheopheresis also became available, at the University of Debrecen, Hungary was the first where it was introduced.

The few larger trials, such as the Trial-Art and RheoNet study reported positive rheological, angiological and ophthalmological effects, which we were also able to observe [10-11]. Wolf at al. have found correlation between retinal microcirculation, plasma viscosity and visual function in patients with macroglobulinemia [23]. Mechanism of action of the treatment at the level of the retina is 

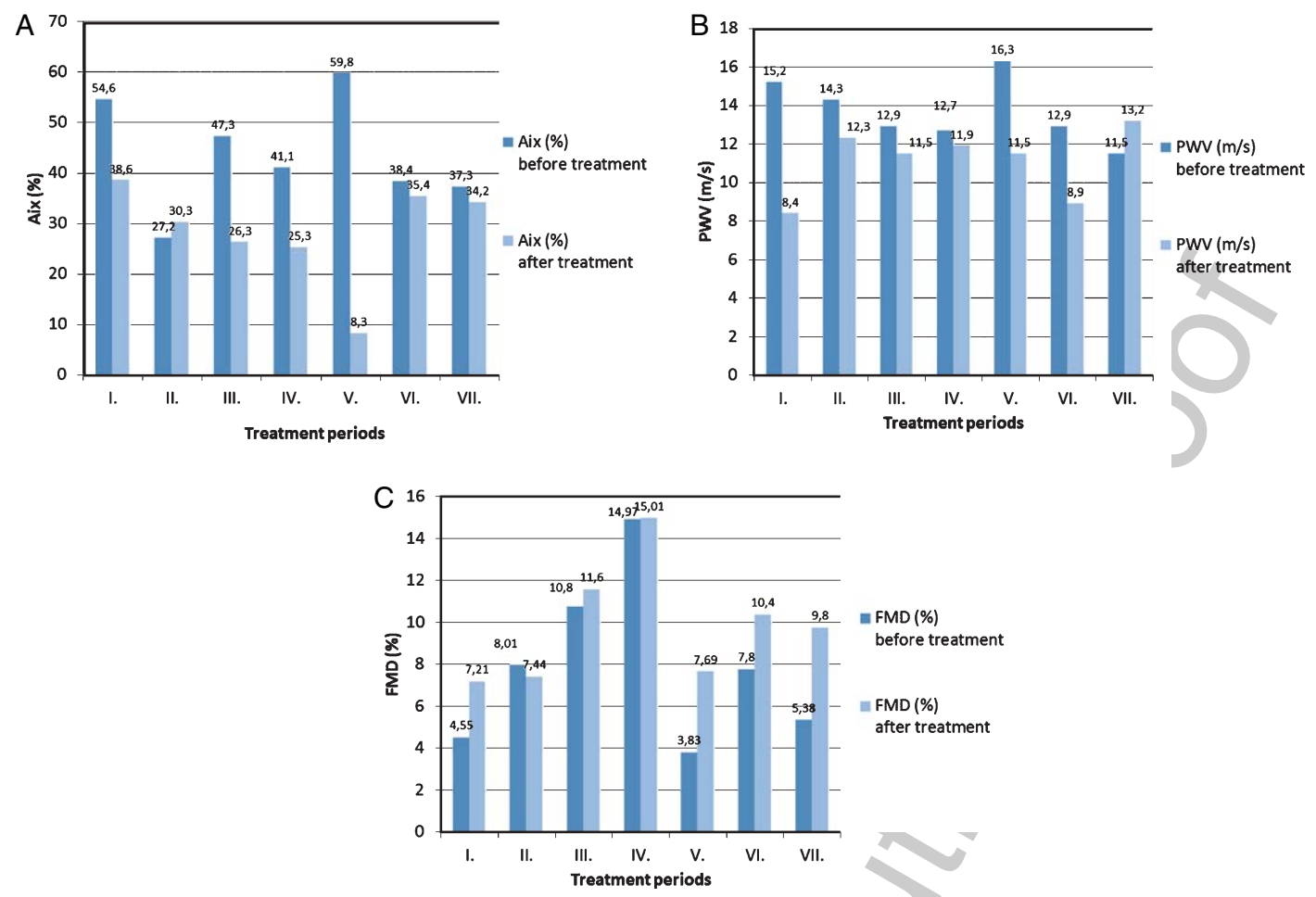

Fig. 3. (a) The effect of rheoferesis treatment on augmentation index, (b) The effect of rheopheresis on pulse wave velocity, (c) The effect of rheopheresis treatment on flow mediated vasodilatation.

probably exhibited via enhancing tight attachment of photoreceptor external/internal segments to the foveal area [18]. It can be especially efficient in such dry AMD forms, like in our case, where pigment epithelium detachment is present with sub-retinal fluid. The latter was completely absorbed by the end of the treatment, and the contour of the fovea was confirmed to be even by OCT. On contrary to this, the almost 10-year-old MIRA study was not able to provide the initially promising achievements on long term, but the selection criteria were not unified [16]. Recently, according to the guidelines of the American Society for Apheresis (ASFA), they recommend rheopheresis as a first choice of treatment in case of dry form of AMD, based on strong evidence specified in a comprehensive evidence-based meta-analysis [21].

Our examinations supported the hypothesis that besides the above mentioned effects of rheopheresis, it may have other vascular and angiological impacts. In line with normalization of viscosity parameters and decreasing the levels of atherogenic lipid fractions, it was proven that rheopheresis has anti-inflammatory effect as well, and it also moderates thrombocyte activation, which has additional beneficial effects too. In addition to all the above mentioned facts, we also found evidence that rheopheresis therapy may improve endothelial functions and stiffness parameters. These effects might have the outmost importance regarding the treatment of vascular diseases developing as a result of atherosclerosis. However, further examinations are needed to discover vascular target points of rheopheresis in details.

\section{Acknowledgments}

"The authors comply with the Ethical Guidelines for Publication in Clinical Hemorheology and Microcirculation as published on the IOS Press website and in Volume 63, 2016, pp. 1-2. of this journal." 


\section{References}

[1] Age-Related Eye Disease Study 2 Research, Group, Lutein + zeaxanthin and omega-3 fatty acids for age-related macular degeneration: The age-related eye disease study 2 (AREDS2) randomized clinical trial, JAMA 309 (2013), 2005-2015.

[2] Age-Related Eye Disease Study Research Group, A randomized, placebo-controlled, clinical trial of high-dose supplementation with vitamins $\mathrm{C}$ and $\mathrm{E}$, beta carotene, and zinc for age-related macular degeneration and vision loss: AREDS report no. 8, Arch Ophthalmol 119 (2001), 1417-1436.

[3] R. Brunner, R.A. Widder, P. Walter, H. Borberg and K. Oette, Change in hemorrheological and biochemical parameters following membrane differential filtration, J Artif Organs 18(12) (1995), 794-798.

[4] R. Brunner, R.A. Widder, P. Walter, C. Lüke, E. Godehardt, K.U. Bartz-Schmidt, K. Heimann and H. Borberg, Influence of membrane differential filtration on the natural course of age-related macular degeneration: A randomized trial, Retina 20(5) (2000), 483-491.

[5] E. Buschini, A.M. Fea, C.A. Lavia, M. Nassisi, G. Pignata, M. Zola and F.M. Grignolo, Recent developments in the management of dry age-related macular degeneration, Clin Ophthalmol 9 (2015), 563-574.

[6] C. Carallo, A. Loprete, G. Mazza, G. Bellotti, M. de Siena, P. Serrao, E.S. Vuoto, M.S. de Franceschi, C. Irace and A. Gnasso, Biphasic hemodynamic effects of LDL-apheresis in common carotid artery, Clin HemorheolMicrocirc 60(3) (2015), 297-307.

[7] Guidelines on the use of therapeutic apheresis in clinical practice-evidence-based approach from the Writing Committee of the American Society for Apheresis: The sixth special issue, J Clin Apher 28(3) (2013),145-284.

[8] M.R. Hardeman, P.T. Goedhart and S. Shin, Methods in hemorheology. In: Handbook of hemorheology and hemodynamics, Baskurt OK, Hardeman MR, Rampling MW, Meiselman HJ, eds. IOS Press, Amsterdam, 2007, pp. 242-266.

[9] R. Klingel, B. Erdtracht, V. Gauss, A. Piazolo, P. Mausfeld-Lafdhiya and C. Diehm, Rheopheresis in patients with critical limb ischemia-results of an open label prospective pilot trial, Ther Apher Dial 9 (2005), 473-481.

[10] R. Klingel, C. Fassbender, A. Heibges, F. Koch, J. Nasemann, K. Engelmann, T. Carl, M. Meinke and B. Erdtracht, RheoNet registry analysis of rheopheresis for microcirculatory disorders with a focus on age-related macular degeneration, Ther Apher Dial 14 (2010), 276-286.

[11] M.J. Koss, P. Kurz, T. Tsobanelis, W. Lehmacher, C. Fassbender, R. Klingel and F.H. Koch, Prospective, randomized, controlled clinical study evaluating the efficacy of Rheopheresis for dry age-related macular degeneration. Dry AMD treatment with Rheopheresis Trial-ART, Graefes Arch Clin Exp Ophthalmol 247 (2009), 1297-1306.

[12] A. Matrai, R.B. Whittington and E. Ernst, A simple method of estimating whole blood viscosity at standardized hematocrit, Clin Hemorheol 7 (1987), 261-265.

[13] K.P. Mellwig, D. Baller, U. Gleichmann, D. Moll, S. Betker, R. Weise and G. Notohamiprodjo, Improvement of coronary vasodilatation capacity through single LDL apheresis, Atherosclerosis 139(1) (1998), 173-178.

[14] S. Michels, P.J. Rosenfeld, C.A. Puliafito, E.N. Marcus and A.S. Venkatraman, Systemic bevacizumab (Avastin) therapy for neovascular age-related macular degeneration twelve-week results of an uncontrolled open-label clini- cal study, Ophthalmology 112 (2005), 1035-1047.

[15] J. Németh, A. Frigyik, O. Vastag, P. Göcze, T. Pető and I. Elek, Vaksági okok Magyarországon 1996 és 2000 között, Szemészet 142 (2005), 127-133.

[16] J.S. Pulido, J.L. Winters and D. Boyer, Preliminary analysis of the final multicenter investigation of rheopheresis for age related macular degeneration (AMD) trial (MIRA-1) results, Trans Am Ophthalmol Soc 104 (2006), $221-231$.

[17] J.S. Pulido, Multicenter Investigation of Rheopheresis for AMD (MIRA-1) Study Group. Multicenter prospective, randomized, double-masked, placebo-controlled study of Rheopheresis to treat nonexudative age-related macular degeneration: Interim analysis, Trans Am Ophthalmol Soc 100 (2002), 85-106; discussion 106-7.

[18] E. Rencová, M. Bláha, J. Studnička, V. Bláha, M. Lánská, O. Renc, A. Stepanov, V. Kratochvílová and H. Langrová, Preservation of the photoreceptor inner/iuter segment junction in dry age-related macular degeneration treated by rheohemapheresis, J Ophthalmol 2015 (2015), 359747.

[19] J. Ringel, MS80 lipoprotein filtration by monet: First clinical application in LDL removal, Atherosclerosis Supplements 11(2) (2010), 126.

[20] P. Schuff-Werner, Extracorporeal hemorheotherapy with selective plasma protein elimination, Jpn, J Apheresis 16 (1997), 25-30.

[21] J. Schwartz, J.L. Winters, A. Padmanabhan, R.A. Balogun, M. Delaney, M.L. Linenberger, Z.M. Szczepiorkowski, M.E. Williams, Y. Wu and B.H. Shaz, Guidelines on the use of therapeutic apheresis in clinical practice-evidence-based approach from the Writing Committee of the American Society for Apheresis: The sixth special issue, J Clin Apher $\mathbf{2 8}$ (2013), 145-284. 
[22] R.A. Widder, R. Brunner and H. Borberg, Changes of haemorheological parameters when using plasma exchange, selective adsorption and membrane differential separation, Transfus Sci 17(4) (1996), 505-510.

[23] S. Wolf, K. Schulte, O. Arend, W.M. Glöckner, S. Handt, F. Jung and M. Reim, Correlation between retinal microcirculation, plasma viscosity and visual function in patients with macroglobulinemia, Clin Hemorheol 12 (1992), 725-731. 